\title{
Atomic jet from SMM1 (FIRS1) in Serpens uncovers protobinary companion
}

\author{
O. Dionatos ${ }^{1,2,3}$, J. K. Jørgensen ${ }^{3,2}$, P. S. Teixeira ${ }^{1}$, M. Güdel ${ }^{1}$, and E. Bergin ${ }^{4}$
}

\author{
1 Department of Astrophysics, University of Vienna, Türkenschanzstrasse 17, 1180 Vienna, Austria \\ e-mail: odysseas.dionatos@univie.ac.at \\ 2 Centre for Star and Planet Formation, Natural History Museum of Denmark, University of Copenhagen, Øster Voldgade 5-7, \\ 1350 Copenhagen K, Denmark \\ 3 Niels Bohr Institute, University of Copenhagen, Juliane Maries Vej 30, 2100 Copenhagen $\varnothing$, Denmark \\ ${ }^{4}$ Department of Astronomy, University of Michigan, 500 Church Street, Ann Arbor MI 48109, USA
}

Received 4 October 2013 / Accepted 17 January 2014

\section{ABSTRACT}

\begin{abstract}
Context. We report on the detection of an atomic jet associated with the protostellar source SMM1 (FIRS1) in Serpens. The morphological and physical characteristics of the atomic jet suggest that there is a more evolved protostellar companion to the Class 0 source SMM1.

Aims. We unravel the molecular and atomic emission around the protostellar source Serpens-SMM1, identify the emission line origin, and assess the evolutionary stage of the driving sources.

Methods. The surroundings of SMM1 were mapped with the Spitzer Infrared Spectrograph (IRS) in slit-scan mode. The complex outflow morphology of the molecular $\left(\mathrm{H}_{2}\right)$ and atomic ([FeII], [NeII], [SiII], [SI]) emission from Spitzer is examined along with deconvolved Spitzer IRAC and MIPS images and high-velocity CO $J=3-2$ outflow maps. The physical conditions of the atomic jet are assessed assuming LTE, non-LTE conditions, and shock models.

Results. The atomic jet is firmly detected in five different [FeII] and [NeII] lines, with possible contributions from [SI] and [SiII]. It is traced very close to SMM1 and peaks at $\sim 5^{\prime \prime}$ from the source at a position angle of $\sim 125^{\circ}$. $\mathrm{H}_{2}$ emission becomes prominent at distances $>5^{\prime \prime}$ from SMM1 and extends at a position angle of $160^{\circ}$. The morphological differences suggest that the atomic emission arises from a companion source, lying in the foreground of the envelope surrounding the embedded protostar SMM1. The molecular and atomic emissions disentangle the large-scale $\mathrm{CO}$ emission into two distinct bipolar outflows, giving further support to a protobinary source. The LTE and non-LTE analysis at the peaks of the [FeII] jet show that emission arises from warm and dense gas $\left(T \sim 1000 \mathrm{~K}, n_{\mathrm{e}} \sim 10^{5}-10^{6} \mathrm{~cm}^{-3}\right)$. These conditions are suggestive of dissociative J-type shocks, and this is further supported by strong water-maser emission observed on the axis of the atomic jet. The mass flux of the jet derived independently for the [FeII] and [NeII] emission is $\sim 10^{7} M_{\odot} \mathrm{yr}^{-1}$, pointing to a more evolved Class I/II protostar as the driving source. Comparisons of the large-scale outflow and atomic jet momentum fluxes show that the latter has adequate thrust to support the CO outflow.

Conclusions. The atomic jet detected by Spitzer for the first time gives the opportunity to disentangle the complex outflow morphology around SMM1 into two precessing outflows. The morphological and physical properties of the outflows reveal that SMM1 is a protobinary source. The momentum flux of the atomic jet indicates that the companion to the deeply embedded Class 0 protostar SMM1 is a more evolved Class I/II source.
\end{abstract}

Key words. stars: formation - stars: jets - ISM: jets and outflows - ISM: atoms - ISM: molecules

\section{Introduction}

The observational manifestations of protostellar ejecta show morphological trends which follow the evolutionary stage of their parent bodies. In young embedded Class 0 protostars, interferometric observations of fast moving gas reveal highly collimated "molecular" jets (e.g. HH211, Gueth \& Guilloteau 1999). Large-scale outflows in embedded (Class 0/I) protostars reflect the interactions between ejecta and the surrounding dense medium. These interactions result in the gradual dispersion of the envelope (Arce \& Sargent 2006), and the protostellar ejecta are eventually observed in the infrared and visual wavelengths as "atomic" jets. Morphological characteristics, such as the high degree of collimation and knotty structure observed in both "molecular" and "atomic" jets suggest a common formation and collimation mechanism (Cabrit 2007). Given that the ejecta in the comparatively more evolved Class II sources are arguably atomic (traced down to a few AU from the protostar, e.g., Agra-Amboage et al. 2011), obscured atomic jets may also be responsible for their molecular counterparts seen in embedded protostars. Indications of the existence of such atomic jets from embedded sources have been found in a few cases (e.g., L1448-mm, Dionatos et al. 2009). However, mid-infrared (midIR) surveys of Class 0 protostars (Lahuis et al. 2010) have detected extended [FeII] and [NeII] emission that could be attributed to jets in only $\sim 10 \%$ of the cases. Atomic lines in the far infrared ([OI] and [CII]), commonly traced around embedded sources (e.g., Green et al. 2013), may be excited by different mechanisms so they cannot be used as safe indicators of atomic jets (Visser et al. 2012). It therefore remains unclear whether molecular jets are manifestations of underlying, obscured atomic jets or represent direct ejecta from embedded protostars (Panoglou et al. 2012).

In addition to their morphological characteristics, protostellar ejecta provide insight into the mass accretion/ejection processes that control protostellar evolution. For embedded sources, it has been shown that the momentum flux, or "thrust", 
of outflows strongly correlates with the luminosity of a protostar, which is directly linked to accretion (Bontemps et al. 1996). Analogous accretion/ejection correlations have been shown to also hold for T Tauri stars (Hartigan et al. 1995). Furthermore, such correlations are found to be valid for both low and high mass protostars, providing evidence for a common formation mechanism independently of the protostellar mass (Wu et al. 2004; Zhang et al. 2005), and provide strong evidence that the accretion rate drops as a protostar evolves. The exact link between accretion and ejection is still debated, however most theoretical models (e.g., X-wind and disk-wind models, Shu et al. 1994; Ferreira 1997, respectivelly) agree that there is a strong link between the momentum output of the jet/wind and the accretion rate. Therefore the jet/outflow mass loss rate and momentum flux can provide indirect measures of the mass accretion rate, and thus constrain the evolutionary stage of a protostar.

Among nearby embedded protostars, SMM1 in Serpens is an outstanding source that has drawn lots of attention over the past 30 years. It is the most prominent source in Serpens $(d=$ 260-415 pc; Straižys et al. 2003; Dzib et al. 2010, respectively, the former value adopted in this work) associated with energetic mass ejection phenomena. Strong, extended outflows traced in a number of molecular and atomic tracers (e.g., Goicoechea et al. 2012; Davis et al. 1999; Testi \& Sargent 1998) co-exist with strong radio and maser emission (e.g., Curiel et al. 1993; van Kempen et al. 2009, respectively). It has been proposed that the complex morphology of the outflows seen at interferometric resolutions (Hogerheijde et al. 1999; Testi \& Sargent 1998) is driven by a protobinary system (White et al. 1995; Dionatos et al. 2010b).

Continuum observations of SMM1 have been the subject of intense scrutiny. Millimeter and submillimeter parts of the spectral energy distribution (SED) reveal an extended envelope and a compact peak corresponding to masses of $8.7 M_{\odot}$ and $0.9 M_{\odot}$, respectively (Hogerheijde et al. 1999). The suggested $M_{\text {env }} / M_{\text {star }}<0.5$ corresponds to a young, deeply embedded Class 0 protostar. Still, the mid-IR part of the spectrum shows significant IRAC emission (Winston et al. 2007), which has been hard to attribute to a Class 0 source. Fitting the full SED, Davis et al. (1999) reported a bolometric temperature of $\sim 38 \mathrm{~K}$ and assigned SMM1 an intermediate evolutionary stage between Classes 0 and I. More recently, Enoch et al. (2009) have modeled SMM1 as a luminous Class 0 source and attributed the near- and mid-IR emission to a massive disk of $\sim 1 M_{\odot}$. Choi (2009), used $\mathrm{mm}$ and $\mathrm{cm}$ continuum interferometric observations, and report on the existence of a weaker secondary continuum source positioned at $\sim 1.8^{\prime \prime}(500 \mathrm{AU})$ to the NW, suggesting that SMM1 is a protostellar binary. From the spectral index between $6.9 \mathrm{~mm}$ and $7 \mathrm{~cm}$, they argue that the secondary peak is a disk source that dominates the IR part of the SED. This was previously suggested based on the mismatch between the positions of the near-IR source EC41 and the millimeter continuum source SMM1 (Eiroa \& Casali 1989; Hodapp 1999; Eiroa et al. 2005). It should be noted that EC41 and SMM1-b are not spatially associated. The possible companion observed in Choi (2009) is also detected in $230 \mathrm{GHz}$ interferometric continuum maps of Enoch et al. (2009), however it was interpreted as a condensation along the outflow.

In this paper we employ Spitzer spectro-imaging observations to disentangle the emission around SMM1. We describe the data reduction in Sect. 2 and discuss the spatial morphology of atomic and molecular $\left(\mathrm{H}_{2}\right.$ and $\left.\mathrm{CO}\right)$ emission lines in Sect. 3. In Sect. 4 we derive the excitation conditions and dynamics of the atomic jet and constrain its possible progenitor. Our conclusions are summarized in Sect. 5.
Table 1. Line intensities at the peaks of emission, extracted for a region equal to the LH spaxel size.

\begin{tabular}{lcrrrc}
\hline \hline \multicolumn{2}{l}{ Element Transition } & $E_{\mathrm{u}}(\mathrm{K})$ & $\lambda(\mu \mathrm{m})$ & $\begin{array}{r}\text { Intensity }\left(10^{-12} \mathrm{~W} \mathrm{~cm}^{-2} \mathrm{sr}^{-1}\right) \\
\text { blue lobe }\end{array}$ & red lobe \\
\hline $\mathrm{H}_{2}$ & $0-0 \mathrm{~S}(2)$ & 1681.76 & 12.2786 & $0.51 \pm 0.08$ & $\ldots$ \\
{$[\mathrm{NeII}]$} & ${ }^{2} \mathrm{P}_{\frac{1}{2}}-{ }^{2} \mathrm{P}_{\frac{3}{2}}$ & 1122.85 & 12.8135 & $2.09 \pm 0.09$ & $0.47 \pm 0.09$ \\
$\mathrm{H}_{2}$ & $0-0 \mathrm{~S}(1)$ & 1015.20 & 17.0348 & $0.41 \pm 0.07$ & $\ldots$ \\
{$[\mathrm{FeII}]$} & ${ }^{4} \mathrm{~F}_{\frac{7}{2}}-{ }^{4} \mathrm{~F}_{\frac{9}{2}}$ & 3496.35 & 17.9359 & $2.82 \pm 0.12$ & $1.24 \pm 0.08$ \\
{$[\mathrm{FeII}]$} & ${ }^{4} \mathrm{~F}_{\frac{5}{2}}-{ }^{4} \mathrm{~F}_{\frac{7}{2}}$ & 4083.1624 .5193 & $3.86 \pm 0.22$ & $\ldots$ \\
{$[\mathrm{SI}]$} & ${ }^{3} \mathrm{P}_{1}-{ }^{3} \mathrm{P}_{2}$ & 569.83 & 25.2490 & $5.28 \pm 0.26$ & $1.92 \pm 0.24$ \\
{$[\mathrm{FeII}]$} & ${ }^{6} \mathrm{D}_{\frac{7}{2}}-{ }^{6} \mathrm{D}_{\frac{9}{2}}$ & 553.62 & 25.9883 & $11.02 \pm 0.37$ & $8.22 \pm 0.18$ \\
{$[\mathrm{SiII}]$} & ${ }^{2} \mathrm{P}_{\frac{3}{2}}-{ }^{2} \mathrm{P}_{\frac{1}{2}}$ & 413.27 & 34.8152 & $12.16 \pm 0.67$ & $9.48 \pm 0.46$ \\
{$[\mathrm{FeII}]$} & ${ }^{6} \mathrm{D}_{\frac{5}{2}}-{ }^{6} \mathrm{D}_{\frac{7}{2}}$ & 960.64 & 35.3487 & $3.34 \pm 0.47$ & $2.31 \pm 0.38$ \\
\hline
\end{tabular}

\section{Data reduction}

The area around SMM1 $\left(\alpha_{J 2000}=18^{\mathrm{h}} 29^{\mathrm{m}} 49.8, \delta_{J 2000}=\right.$ $+01^{\mathrm{d}} 15^{\mathrm{m}} 20^{\mathrm{m}}$. , Choi 2009) was observed on June 5, 2009 as part of the "Searching for the Missing Sulfur in the Dense ISM" program (E. Bergin, P.I.). The short-high (SH) and long-high (LH) modules of the Spitzer Infrared Spectrograph (IRS, Houck et al. 2004) were employed, providing a wavelength coverage between 10 and $37 \mu \mathrm{m}$ at a resolution of $R \sim 600$. Observations were performed in slit-scan mode consisting of consecutive integrations after shifting the slit to the parallel and perpendicular directions relative to the slit length, until the desired area is covered. The $\mathrm{SH}$ and $\mathrm{LH}$ scans consist of grids of $6 \times 21$ and $11 \times 10$ observations, respectively, centered at $\alpha_{J 2000}=$ $18^{\mathrm{h}} 29^{\mathrm{m}} 48^{\mathrm{s}} .7, \delta_{J 2000}=+01^{\mathrm{d}} 15^{\mathrm{m}} 10^{\mathrm{s}} .4$. The integration time per pointing is 30 and $6 \mathrm{~s}$ for the $\mathrm{SH}$ and LH modules, respectively.

Spectra were retrieved from the Spitzer Heritage Archive (SHA). Initial data processing was performed with version S18.7 of the Spitzer Science Center pipeline. Spectral data cubes were compiled using the CUBISM software (Smith et al. 2007). $\mathrm{Bad} / \mathrm{rogue}$ pixels were removed using dedicated off-target observations. Emission line maps were reconstructed through customized procedures. In these, for each spatial pixel (or spaxel) of a datacube, the flux for each spectral line of interest was calculated by fitting a Gaussian after subtracting a local first- or second-order polynomial baseline. The resulting line intensity maps for the IRS data have a square spaxel of side equal to the width of the high resolution IRS modules (4.7" for SH and 11.1" for LH), while the instrumental point spread function of Spitzer ranges between $\sim 3^{\prime \prime}$ at $10 \mu \mathrm{m}$ to $11^{\prime \prime}$ at $38 \mu \mathrm{m}$.

The wavelength range covered by the $\mathrm{SH}$ and $\mathrm{LH}$ modules is limited toward the shortend to $10 \mu \mathrm{m}$. As a result, the higher energy rotational $\mathrm{H}_{2}$ transitions $(\mathrm{S}(3)-\mathrm{S}(7))$ and other possible atomic lines are not included in the current data. However, lower energy $\mathrm{H}_{2}$ and atomic lines, mainly from [FeII] but also from $[\mathrm{NeII}],[\mathrm{SI}]$, and [SiII] are detected (Table 1), indicating a very energetic environment.

\section{Outflow morphology}

The upper panel of Fig. 1 presents the spatial distribution of the $[\mathrm{NeII}]{ }^{2} \mathrm{P}_{\frac{1}{2}}-{ }^{2} \mathrm{P}_{\frac{3}{2}}$ transition at $12.8 \mu \mathrm{m}$ along with the observed emission distribution from the $\mathrm{H}_{2} \mathrm{~S}(2)$ line centered at $12.3 \mu \mathrm{m}$. The lower panel of the same figure shows the emission pattern from [FeII] ${ }^{4} \mathrm{~F}_{\frac{7}{2}}-{ }^{4} \mathrm{~F}_{\frac{9}{2}}$ and $\mathrm{H}_{2} \mathrm{~S}(1)$ lines, centered at 18 and $17 \mu \mathrm{m}$, respectively. All lines detected with the $\mathrm{SH}$ module are superimposed on an IRAC band- 2 image, centered at $4.5 \mu \mathrm{m}$. 
O. Dionatos et al.: Atomic jet from SMM1 (FIRS1) in Serpens uncovers protobinary companion
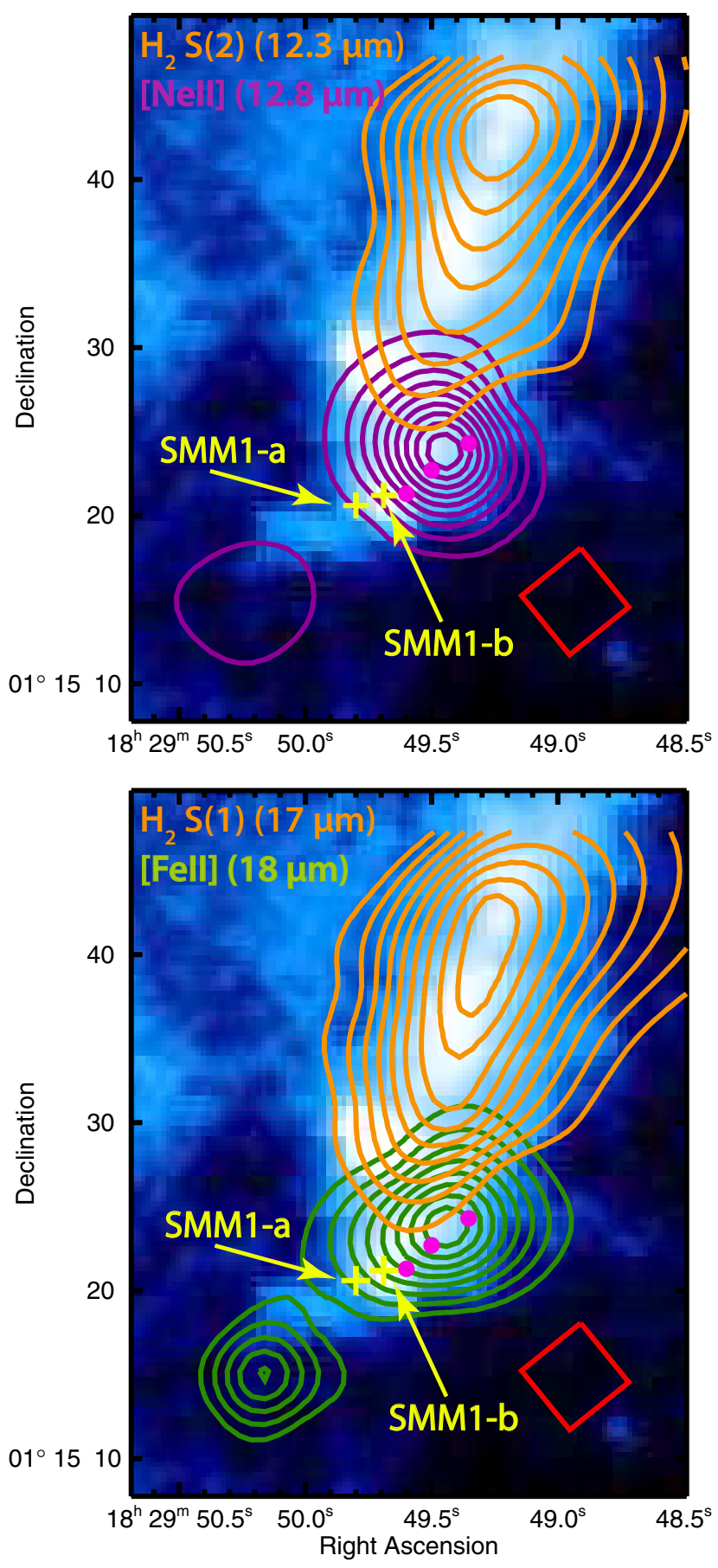

Fig. 1. Emission line maps of the $[\mathrm{NeII}]{ }^{2} \mathrm{P}_{\frac{1}{2}}-{ }^{2} \mathrm{P}_{\frac{3}{2}}$ and $\mathrm{H}_{2} \mathrm{~S}(2)$ lines (top panel) and the $[\mathrm{Fe} \mathrm{II}]{ }^{4} \mathrm{~F}_{\frac{7}{2}}-{ }^{4} \mathrm{~F}_{\frac{9}{2}}$ and $\mathrm{H}_{2} \mathrm{~S}(1)$ lines (lower panel). On both panels line emission is superimposed on an IRAC band-2 $(4.5 \mu \mathrm{m})$ image. The positions of SMM1-a and SMM1-b (to the SE and NW, with respect to each other) from Choi (2009) are marked with crosses, and the SH spaxel size and orientation is marked as a (red) square in the lower right corner of each panel. The (magenta) filled circles show the positions of the $163 \mathrm{GHz} \mathrm{H} \mathrm{H}_{2} \mathrm{O}$ maser emission (van Kempen et al. 2009), coincident with the NW lobe of the atomic emission. Contour levels are at $10-90 \%$ of the peak emission for each transition, as listed in Table 1.

The positions of $6.9 \mathrm{~cm}$ continuum sources SMM1-a (to the SE) and SMM1-b (to the NW) from Choi (2009), along with the locations of $183 \mathrm{GHz} \mathrm{H} \mathrm{H}_{2} \mathrm{O}$ maser hotspots from van Kempen et al. (2009), are also indicated.

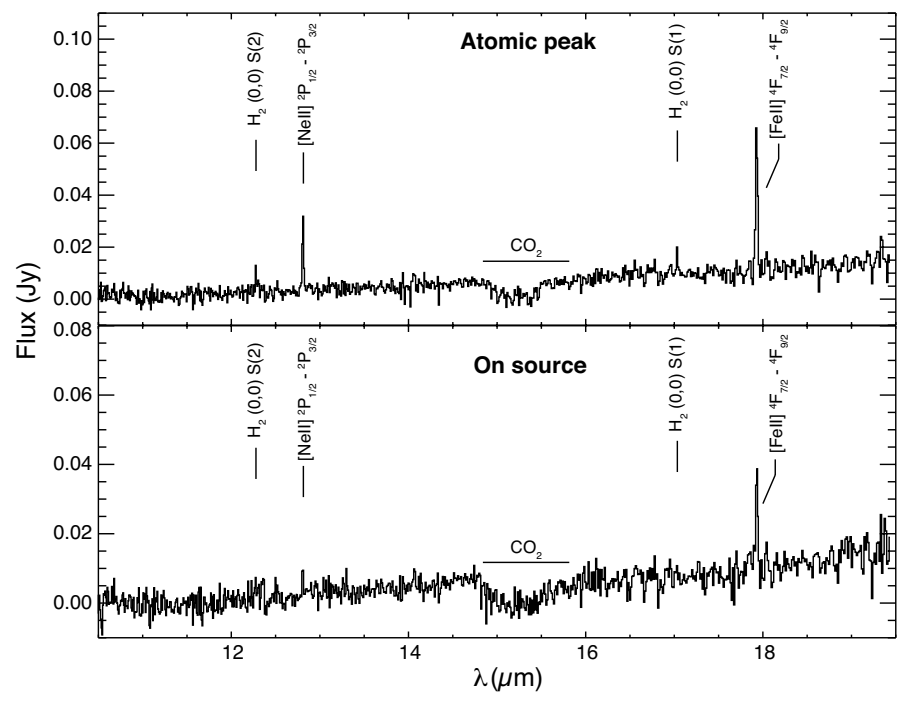

Fig. 2. IRS spectra from the SH module (10-20 $\mu \mathrm{m})$ corresponding to the spaxels encompassing the on-source and atomic peak positions in Fig. 1 (lower and upper panels, respectively). Both positions are dominated by strong atomic emission lines. $\mathrm{No}_{2}$ emission is detected at the on-source spaxel. $\mathrm{CO}_{2}$ ice absorption bands are prominent in both spectra, indicating extended continuum emission to angular distances $>5 "$ and a foreground absorbing layer.

The $\mathrm{H}_{2}$ line maps show very similar morphologies following the bright ridge recorded in the IRAC image at a position angle of $\sim 340^{\circ}$. The $\mathrm{H}_{2}$ emission becomes apparent at angular distances $>5^{\prime \prime}$ and peaks at $\sim 20^{\prime \prime}$ from the position of SMM1-a. The [NeII] and [FeII] lines are detected on the positions of SMM1-a and SMM1-b and exhibit two symmetric lobes extending to the NW and SE, peaking at distances of $\sim 5^{\prime \prime}$. The strongest peak to the NW from the centimeter sources is aligned with the positions of $\mathrm{H}_{2} \mathrm{O}$ maser detections. The atomic emission extends along a position angle of $125^{\circ}$, which is in roughly the same direction but still different from the one traced by $\mathrm{H}_{2}$. Figure 2 presents IRS/SH spectra in the range between $10 \mu \mathrm{m}$ and $20 \mu \mathrm{m}$ for the spaxels pertaining to the source and atomic emission maxima positions (lower and upper panels, respectively). At the onsource position, the [FeII] and [NeII] lines are prominent, however no $\mathrm{H}_{2}$ is detected. At the position where the atomic lines peak, $\mathrm{H}_{2}$ lines are detected, but they are weaker than the atomic ones. The $\mathrm{CO}_{2}$ ice absorption band at $\sim 15 \mu \mathrm{m}$ is prominent in both positions. This band appears superimposed on continuum, suggesting that the continuum emission extends up to $\sim 5$ " or more from SMM1. The $\mathrm{CO}_{2}$ band likely originates in ice mantles on dust grains, located in a foreground layer (see also Sect. 4).

In Fig. 3, we present the emission pattern of the atomic lines detected with the LH module. The upper panels show the [FeII] transitions at 26, 24.5, and $35.4 \mu \mathrm{m}$. The emission pattern of all [FeII] lines shows a very similar morphology to the pattern traced by the [FeII] $18 \mu \mathrm{m}$, despite the inferior angular resolution of the LH compared to the SH module. The [FeII] lines are detected very close to the continuum sources, and a strong peak towards the NW at $\sim 5^{\prime \prime}$ from SMM1 is detected in all cases. The weaker peak at the SE is not resolved in the $26 \mu \mathrm{m}$ and $35.4 \mu \mathrm{m}$ maps, which do, however, preserve the overall morphology of the $18 \mu \mathrm{m}$ [FeII] line. The $24.5 \mu \mathrm{m}$ [FeII] emission is not resolved at the SE peak. The [SI] and [SiII] lines presented in the lower panels of Fig. 3 peak around the same location as the [FeII] lines, but also show elongated structures to the NW with a closer resemblance to the $\mathrm{H}_{2}$. 


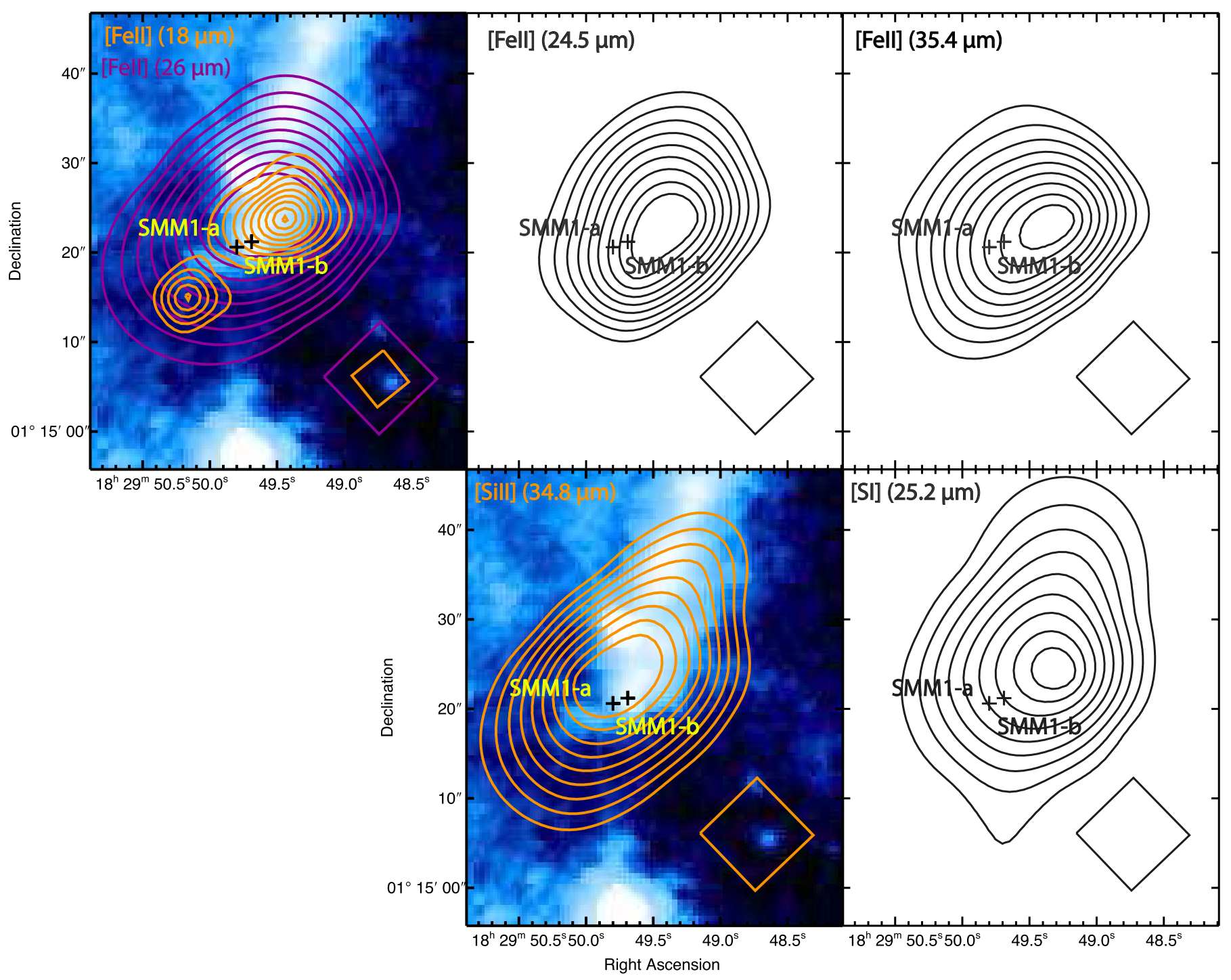

Fig. 3. Spectral maps of atomic lines detected with the LH module: upper panels show the distribution of [FeII] lines and lower panels the morphology of [SiII] and [SI]. The contour maps in the leftmost panels are superimposed on an IRAC band-2 image. The LH and SH module spaxel size and orientation are presented at the lower right corner of each panel. The [FeII] $18 \mu$ m morphology mapped with the SH module (Fig. 1) is presented as reference in the upper left panel along with the $26 \mu \mathrm{m}$ [FeII] line. Even thought the LH footprint covers more area than the $\mathrm{SH}$ one by a factor of 4 , [FeII] lines show a consistent morphology. In contrast, [SiII] and [SI] follow closer the $\mathrm{H}_{2}$ emission pattern also traced by the bright ridge in the IRAC image. Contour levels are at 10-90\% of the peak emission for each transition, listed in Table 1

The extended emission seen in the Spitzer/MIPS Mosaic image at $24 \mu \mathrm{m}$ (Fig. 4) is dominated by the [SI] and [FeII] lines encompassed within the band (Velusamy et al. 2007, 2011). At an angular resolution of $5^{\prime \prime}$, the Mosaic image is in excellent agreement with the line-map morphologies presented in Fig. 3. Deconvolution methods, such as the HiRes technique (e.g., Velusamy et al. 2008, 2014), can enhance the angular resolution of Spitzer images. The HIRes $24 \mu \mathrm{m}$ image presented in Fig. 4 (Velusamy et al. 2014) reaches a resolution of $\sim 2^{\prime \prime}$; on this scale, the extended atomic emission is directly comparable to the $[\mathrm{FeII}]$ and $[\mathrm{NeII}]$ line morphologies seen in the SH maps of Fig. 1. The HiRes IRAC image at $4.5 \mu \mathrm{m}$ in Fig. 4 at a resolution of $\sim 0.8^{\prime \prime}$ (Velusamy et al. 2014) is dominated by highly excited $\mathrm{H}_{2}$ and continuum emission from more evolved protostars. The HiRes technique reveals a number of structures that are not discernible in the IRAC image presented in Fig. 1: SMM1-b is coincident with bright spot of emission, and the nearIR source EC-41 (e.g., Hodapp 1999) becomes apparent at 5" to the north. A secondary bright spot to the NW of SMM1-b is likely related to the $6.9 \mathrm{~mm}$ knot-E in Choi (2009). The high resolving power reached by the HiRes deconvolution method reveals emission structures in the Spitzer images that are in excellent agreement to the ones traced in the IRS line maps and the interferometric observations of Choi (2009).

The emission morphology traced by the [FeII] and [NeII] lines and the $24 \mu \mathrm{m}$ HiRes image have the characteristics of a jet. To the resolution available, the emission is well aligned and confined into a single row of spaxels. It is detected very close to the protostellar sources, and terminated at the emission maxima. These positions likely correspond to terminating bow shocks, as is commonly observed in protostellar jets (e.g., HH211, Dionatos et al. 2010a). However, the origin of the emission is not clear. It may represent either intrinsic atomic ejecta, as observed in evolved young stellar objects, emission produced in small shocks along the jet propagation axis, or both.

The morphologies of the atomic jet and the $\mathrm{H}_{2}$ emission suggest that the former represents the intrinsic ejecta from the embedded protostar, SMM1, carving out a cavity on the protostellar 

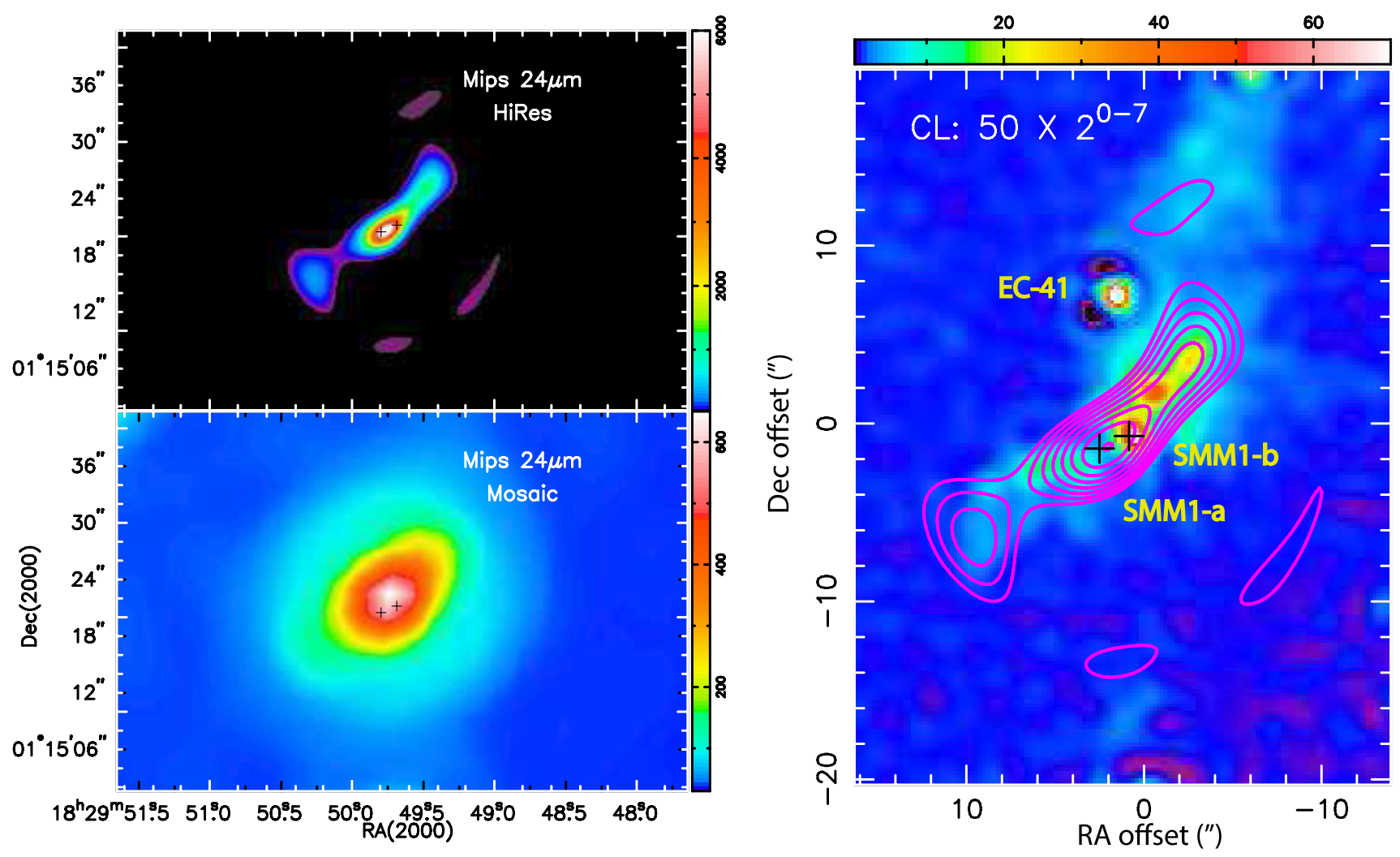

Fig. 4. Left: MIPS $24 \mu \mathrm{m}$ mosaic image before and after HiRes deconvolution (lower and upper panels, respectively). The morphology delineated in the mosaic image at a resolution of $5^{\prime \prime}$ is in excellent agreement with the LH module spectral line maps shown in Fig. 3. The HiRes MIPS image reaching a resolution of 2", compares directly to the atomic jet traced in the SH line maps (Fig. 1). Right: MIPS HiRes image (purple contours) superimposed on an IRAC $4.5 \mu \mathrm{m}$ HiRes image. The IRAC image is dominated by highly excited $\mathrm{H}_{2}$ emission and is in good agreement with the morphology of the atomic jet seen in the MIPS HiRes band. The companion source SMM1-b is coincident with a bright spot of emission in the IRAC channel, while the Class 0 source SMM1-a shows no association with the emission in the IRAC band. Images provided by T. Velusamy, priv. comm. (Velusamy et al. 2014).

envelope, which is delineated by the $\mathrm{H}_{2}$ emission. The undetected, symmetric cavity walls to the south in the NW lobe could be attributed to a steep gradient in the local density in the $\mathrm{N}-\mathrm{S}$ direction. Such a description is compatible with the interpretation of the $\mathrm{HCO}^{+}$and $\mathrm{HCN} J=1-0$ interferometric maps of Hogerheijde et al. (1999). Offsets between the atomic and $\mathrm{H}_{2}$ emission have also been observed in HH54 (Neufeld et al. 2006), since the two sets of lines trace very different excitation conditions in shocks. However, the offsets observed in HH54 lie along the propagation axis of the protostellar jet.

In the present data, the excitation of $\mathrm{H}_{2}$ and the atomic lines occurs on two different axes, and the molecular and atomic components are not cospatial. Given that the pairs of atomic and $\mathrm{H}_{2}$ lines presented in Fig. 1 are separated by $\sim 1 \mu \mathrm{m}$ or less in wavelength, each pair of lines should be equally affected by extinction. The fact that the atomic jet is traced down to the protostellar source suggests it is less extinct than the $\mathrm{H}_{2}$ emission. Close to embedded protostars, mid-IR emission is often highly extinct due to the dense envelope (e.g., Dionatos et al. 2009), so the atomic emission most likely originates in a region outside of the envelope and in the foreground of the Class 0 protostar SMM1-a. Given the different position angles and extents of the low- $J \mathrm{H}_{2}$ and atomic components, the companion source SMM1-b suggested by Choi (2009) is a strong candidate as the driving source. It is aligned well with the atomic jet, and as a more evolved source, it is expected to drive an atomic jet, as discussed in Sect. 4. SMM1-b shows strong emission in the deconvolved HiResIRAC image (Fig. 4) in support of its classification as an evolved protostar.
The radio jet (Choi 2009; Curiel et al. 1993) and maser emission (van Kempen et al. 2009) associated with SMM1 extend in the same direction as the atomic jet at a position angle of $\sim 135^{\circ}$. Choi (2009) associates the radio jet with the source SMM1-a and the $\mathrm{H}_{2}$ emission with SMM1-b, even thoughin their high resolution maps, SMM1-b lies on the radio jet axis and SMM1-a is offset by $\sim 1^{\prime \prime}$ to the NE. The morphological evidence presented here and the analysis in the following paragraphs suggest that SMM1-a is responsible for the low- $J \mathrm{H}_{2}$ emission, and SMM1-b for the atomic and radio jets.

To examine the $\mathrm{H}_{2}$ and atomic emission morphology further, we employ the CO $J=3-2$ data of the SMM1 region from Dionatos et al. (2010b) observed with JCMT. In the lefthand panel of Fig. 5, we present the high velocity blue- and redshifted CO emission, overlaid on the IRAC band- 2 image. The CO maps display a complex morphology, especially towards the NW, where entangled blue- and red-shifted emission is seen extending in the same direction. The red-shifted lobe, $\mathrm{Br} 2$ (following the nomenclature of Dionatos et al. 2010b), is weaker and peaks closer to SMM1 than the blue-shifted lobe, Bb1. Strong red-shifted emission extends to the SE (lobe Br1) and blueshifted gas directly to the $\mathrm{S}(\mathrm{Bb} 2)$. As noted in Dionatos et al. (2010b), the pattern of the CO line wings for Bb1 and Br1 shows signatures of high velocity "bullets", which are not observed towards the peaks of $\mathrm{Bb} 2$ and $\mathrm{Br} 2$. This characteristic provides a clear association between lobes $\mathrm{Bb} 1$ and $\mathrm{Br} 1$ in a bipolar outflow scheme and differentiates them from the $\mathrm{Bb} 2-\mathrm{Br} 2$ pair.

The middle panel of Fig. 5 presents the $17 \mu \mathrm{m} \mathrm{H}_{2}$ and the $18 \mu \mathrm{m}$ [FeII] line maps, along with the $\mathrm{CO}$ outflow emission. 


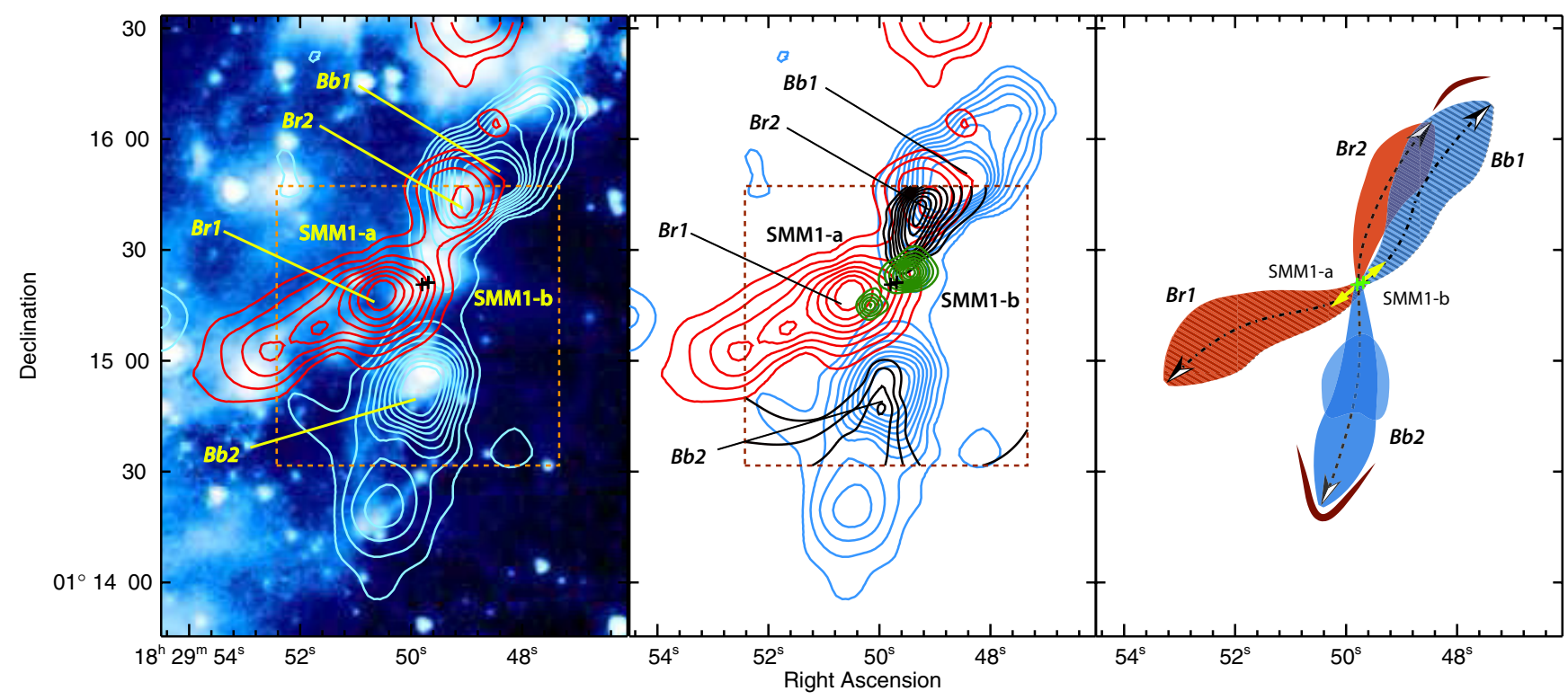

Fig. 5. Left: high velocity CO $J=3-2$ blue- and red-shifted emission (integrated over $v_{\text {lsr }}$ ranges of $[-30,0] \mathrm{km} \mathrm{s}^{-1}$ and $\left[18\right.$, 48] km s${ }^{-1}$, rest $v_{\mathrm{lsr}}=8.8 \mathrm{~km} \mathrm{~s}^{-1}$, and indicated as light blue and red solid contours, respectively) around SMM1 superimposed on an IRAC band-2 image. Outflow components are indicated using the nomenclature of Dionatos et al. (2010b) along with the positions of protostellar sources associated with the outflows. The dashed box delineates the limits of the LH Spitzer maps. Center: CO $J=3-2$ emission as in the left panel, along with the $\mathrm{H}_{2} \mathrm{~S}(1)$ (black) and [FeII] $18 \mu \mathrm{m}$ (green) line maps. Notice the dimensions of the image in comparison to the details presented in Figs. 1 and 2. $\mathrm{H}_{2}$ emission follows well the pattern of $\mathrm{CO}$ lobes $\mathrm{Br} 2$ and $\mathrm{Bb} 2$ in a N-S orientation, whereas the [FeII] emission, despite its shorter extent, has the same orientation as the Bb1-Br1 CO lobes pointing to the SE-NW direction. Right: Sketch of the proposed outflow structure from SMM1-a and b. The outflow driven by the embedded source (SMM1-a) has an S-shaped pattern extending roughly in the N-S axis indicated with a dashed line and solid colors. The same outflow is most likely associated with the bow-shocks visible in the Spitzer image of the left panel, delineated here as dark red $V$-shaped structures. The butterfly-like outflow extending in the SE-NW direction (delineated with a dash-dotted line and hatched colors) most likely corresponds to the less embedded, foreground protostar (SMM1-b). The yellow arrows at the base of the outflows show the direction of the atomic jet.

It becomes apparent that the $\mathrm{H}_{2}$ emission is coincident with the $\mathrm{CO}$ lobes, $\mathrm{Bb} 2$ and $\mathrm{Br} 2$, in an almost $\mathrm{N}-\mathrm{S}$ orientation. [FeII] emission is located at the base of the outflow complex and has practically the same orientation as the bipolar outflow Bb1$\mathrm{Br} 1$, extending from the NW to the SE. The strongest atomic peak at the NW is associated with the blue-shifted CO lobe Bb1 and the weaker atomic lobe observed at the SE with the redshifted lobe Br1. The latter lobe is moving inwards the cloud to regions of higher extinction, which is consistent with the weaker atomic emission to the SE. The bright ridge to the NW seen in the IRAC image corresponds to the superposition of the $\mathrm{Br} 2$ and $\mathrm{Bb} 1$ outflows, which are driven by two different protostellar sources.

The right-hand panel of Fig. 5 shows a sketch of the proposed scenario. The red-shifted emission to the $\mathrm{N}(\mathrm{Br} 2)$ is associated with the lobe $\mathrm{Bb} 2$ to the $\mathrm{S}$ (solid color lobes), as indicated by the $\mathrm{H}_{2}$ emission, and is most likely driven by the embedded protostellar source SMM1-a. The bipolar outflow Bb1-Br1 exhibiting a "bullet" structure in CO is associated with the atomic jet and is most likely driven by the less embedded protostar SMM1-b, which lies in the foreground. The binary source scenario can therefore disentangle the complex outflow morphology observed in $\mathrm{CO}$, and is further supported by the excitation and dynamical properties of the [FeII] lines discussed in the next section.

\section{Analysis and discussion}

To assess the origin of the atomic emission and its possible progenitor, in the following sections we attempt to constrain its excitation conditions following both local thermodynamic equilibrium (LTE) and non-LTE approaches.

\subsection{LTE analysis}

Excitation diagrams are representations of the column density normalized for the upper level degeneracy of a transition and plotted against the corresponding upper level energy of that transition. These diagrams provide a simple but powerful tool for examining the excitation of gas, assuming optically thin emission and LTE conditions. As long as these assumptions are valid, excitation diagrams can readily provide the excitation temperature and column density of the species under consideration (Goldsmith \& Langer 1999).

The excitation diagram method is commonly used for analyzing molecular emission (e.g., Neufeld et al. 2009) and is employed here for the study of atomic lines. The [FeII] lines arise from forbidden transitions with Einstein coefficients for spontaneous de-excitation in the order of $10^{-30} \mathrm{~s}^{-1}$, and thus are optically thin. Radiative rates, level energies, and degeneracies for [FeII] were retrieved from Wiese \& Fuhr (2007) using the Atomic Spectra Database of the National Institute of Standards and Technology ${ }^{1}$ (NIST- ASD, Ralchenko et al. 2011).

In Fig. 6, we present the excitation diagrams for the NW and SE peaks of [FeII], sampled at the LH pixel scale (see Table 1). From the slope of the fitted lines, derived temperatures range between 1000-1600 K, with the higher values being observed towards the NW. The slope of the fit at the NW position is strongly affected by the $24.5 \mu \mathrm{m}$ line intensity (at $E_{\mathrm{u}} \sim 4000 \mathrm{~K}$ ), which may be enhanced by residual bad pixel contamination. If ignored, the temperature for the two positions is consistent at $\sim 1000 \mathrm{~K}$. In excitation diagrams, column densities can be estimated from the intercept of the fitted line with the ordinate,

1 http://www.nist.gov/index.html 
O. Dionatos et al.: Atomic jet from SMM1 (FIRS1) in Serpens uncovers protobinary companion

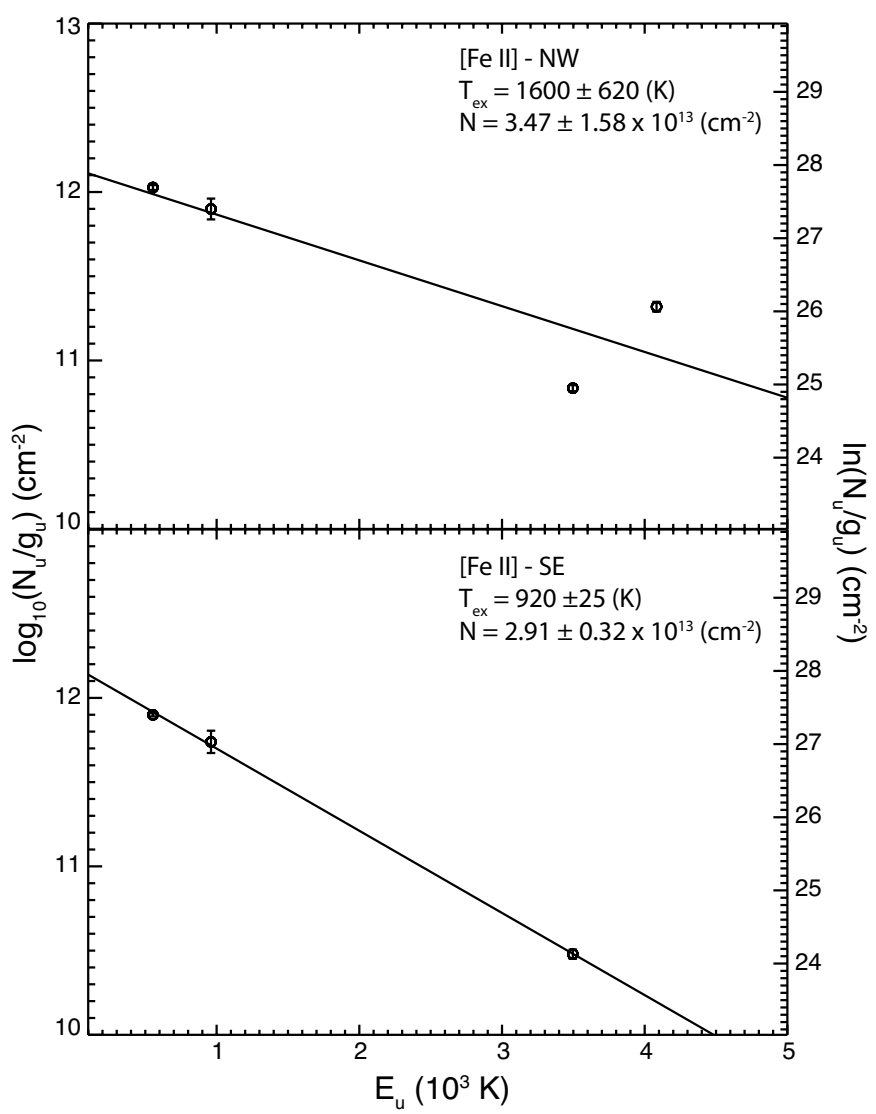

Fig. 6. [FeII] excitation diagram for the NW and SE outflow lobes (upper and lower panels, respectively). Derived temperatures and column densities are reported in the upper right corner of each panel. The $24.5 \mu \mathrm{m}$ line intensity at $E_{\mathrm{u}} \sim 4000 \mathrm{~K}$ is likely affected by residual bad pixel contamination; if excluded from the fit, temperatures at both positions are at $\sim 1000 \mathrm{~K}$.

given that the partition function at a given temperature is known. For the column density calculations in this work, we have employed the partition function values from NIST-ASD for the estimated range of temperatures. Derived column densities are $\sim 3 \times 10^{13} \mathrm{~cm}^{-2}$ for the area covered by the LH spaxel. However, the emitting area of [FeII] is likely smaller, so the present values are probably beam-diluted and therefore represent lower limits.

\subsection{Non-LTE}

For the non-LTE analysis of the [FeII] emission, we employed the statistical equilibrium radiative transfer code, RADEX (van der Tak et al. 2007). As in the case of LTE, we retrieved radiative rates, level energies, and degeneracies from NISTASD. With a first estimate of the excitation conditions from the LTE analysis, we considered the excitation of iron through collisions with electrons. Collisions with atomic hydrogen become important only at much lower temperatures and densities (see Dionatos et al. 2009). Electron collisional rates were retrieved from the TIPbase of the IRON project ${ }^{2}$, based on the calculations of Zhang \& Pradhan (1995).

We ran RADEX for a grid of temperatures ranging from 1000 to $5000 \mathrm{~K}$ and densities between $10^{3}$ and $10^{6} \mathrm{~cm}^{-3}$. The

\footnotetext{
2 http://www.usm.uni-muenchen.de/people/ip/ iron-project.html
}

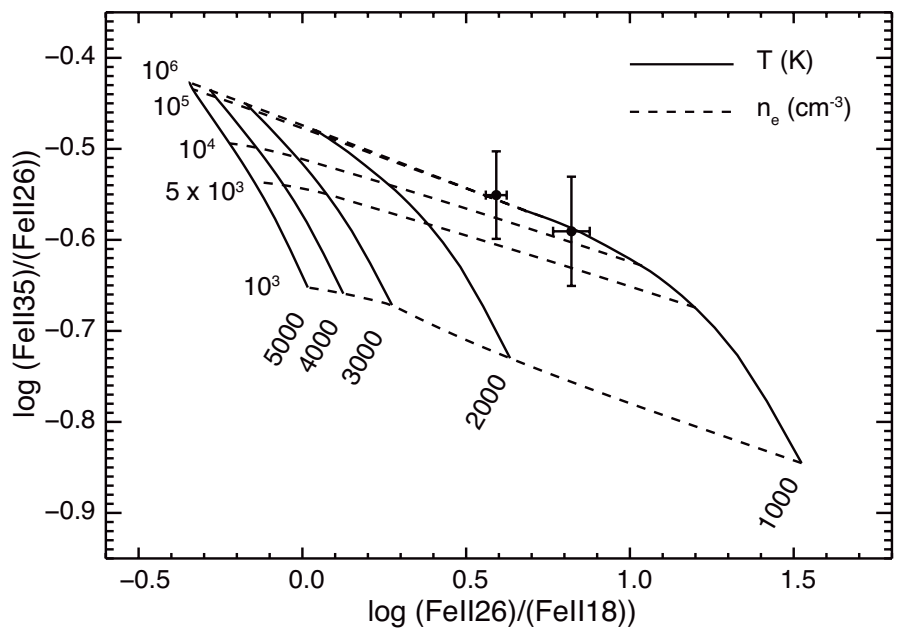

Fig. 7. Non-LTE diagnostic diagram of the [FeII] emission for collisions with electrons created with RADEX. The temperature-sensitive $26 / 18 \mu \mathrm{m}$ ratio (solid lines) is plotted against the electron densitysensitive $35 / 26 \mu \mathrm{m}$ ratio (dashed lines). The observed ratios (filled circles with error bars) indicate $T \sim 1000 \mathrm{~K}$ and $n_{\mathrm{e}} \sim 10^{5}-10^{6} \mathrm{~cm}^{-3}$.

grid results are presented in Fig. 7 in the form of line ratios. We employ the $26 \mu \mathrm{m}$ over the $18 \mu \mathrm{m}$ ratio as a temperature probe, since these lines arise from levels that are separated well in excitation, so that their population depends mainly on the efficiency of the excitation process (collisions with electrons in the current case). In addition, the ratio of the $34.5 \mu \mathrm{m}$ over the $26 \mu \mathrm{m}$ lines is sensitive to density, since these lines arise from levels close in excitation energy, but they have different Einstein coefficients for spontaneous emission. The $24.5 \mu \mathrm{m}$ to $18 \mu \mathrm{m}$ ratio could also be used as a density probe; however, the $24.5 \mu \mathrm{m}$ line is detected at a single position, and the LTE analysis has shown that it may be unreliable.

The observed line ratios in Fig. 7 lie at temperatures of $1000-1200 \mathrm{~K}$. This is in excellent agreement to the temperatures derived from the LTE analysis. The electron densities derived lie between $10^{5}$ and $10^{6} \mathrm{~cm}^{-3}$, even though values as low as $5 \times 10^{3}$ are implied by the high uncertainties of the ratio associated mostly to the uncertainties of the $34.5 \mu \mathrm{m}$ line flux levels.

\subsection{Excitation of the atomic gas}

Derived temperatures at the atomic jet peaks are consistent for both the LTE and non-LTE analysis, and lie at about $\sim 1000 \mathrm{~K}$. This corresponds to "hot" gas for embedded sources, as derived in the mid-IR mainly from $\mathrm{H}_{2}$ emission (Lahuis et al. 2010). The $\mathrm{H}_{2}$ emission in the line maps observed at the atomic peaks may be generated by the same processes, and the higher $J-\mathrm{H}_{2}$ transitions in the HiRes IRAC images show that the atomic and $\mathrm{H}_{2}$ components are spatially coincident. However, the lack of higher $J-\mathrm{H}_{2}$ line maps does not allow us to directly compare the excitation conditions between the atomic and the hot $\mathrm{H}_{2}$ components. In a broader sample of 43 embedded sources observed with Spitzer/IRS (Lahuis et al. 2010), only four display extended [FeII] $18 \mu \mathrm{m}$ emission, which is indicative of highly excited atomic gas. Interestingly, two of them (SMM3 and SMM4) are also located in the Serpens molecular cloud. For all these sources, a hot $\mathrm{H}_{2}$ component is also present ${ }^{3}$.

3 Lahuis et al. (2010) report no hot component for SMM3 and SMM4 in Serpens, but this is detected in Dionatos et al. (2013). 
Electron densities between $10^{5}$ and $10^{6} \mathrm{~cm}^{-3}$ are well above the levels of $500-1000 \mathrm{~cm}^{-3}$ estimated in the case of L1448$\mathrm{mm}$, where no $18 \mu \mathrm{m}$ [FeII] lines were detected (Dionatos et al. 2009). The electron densities derived from the non-LTE analysis are consistent with the values typically observed in "atomic" jets from Class I protostars (Nisini et al. 2002). However, typical temperatures for "atomic" jets range between 7000 and $15000 \mathrm{~K}$ (Takami et al. 2004), which is much higher than the $\sim 1000 \mathrm{~K}$ derived by our analysis. The near-IR [FeII] lines, however, arise from transitions of much higher energies and may as well correspond to a more energetic component of the jet or the shocks along its axis of propagation. Narrow-band imaging for the typical "atomic" jet tracer [SII] $\lambda \lambda 6716,6731 \AA$ (Davis et al. 1999) does not detect any optical jet counterpart, indicating that the atomic jet observed here stands behind a layer of dust that obscures it in the visual bands. This is also suggested by the detection of extended $\mathrm{CO}_{2}$ absorption bands also seen in the outflow positions (Fig. 2) and the extended continuum ridge lying across the atomic jet propagation axis as seen in the interferometric maps of Enoch et al. (2009). The same absorbing layer also obscures SMM1-a which lies in the background of SMM1-b (Sect. 3).

The positioning of both sources behind the absorbing layer suggests that they are probably lying at similar distances from the observer. In addition, their angular separation of $\sim 1.5^{\prime \prime}$ indicates that they are gravitationally bound, forming a protobinary system. Concerning the evolutionary stage of the sources, it is well established that SMM1-a is a deeply embedded Class 0 protostar (e.g., Larsson et al. 2000). This is also supported by the HiRes images of Velusamy et al. (2014), showing emission at $24 \mu \mathrm{m}$ but not at $4.5 \mu \mathrm{m}$. In stark contrast, SMM1-b is coincident with a bright point source at $4.5 \mu \mathrm{m}$, which supports its classification by Choi (2009) as an evolved source. In conclusion, the current observational data suggest that the SMM1-a/ SMM1-b system is a protobinary.

Discussing the outflow morphology in Sect. 3, we concluded that the atomic jet is most likely associated with the high velocity "bullet" CO gas, reaching radial velocities as high as $50 \mathrm{~km} \mathrm{~s}^{-1}$. Yildiz et al. (2013) demonstrate that the velocity traced by low- $J$ $\mathrm{CO}$ lines is only a lower limit since higher $J$ transitions show increasingly higher velocity wings. The high velocity of the material associated with the atomic emission has also been suggested by Goicoechea et al. (2012), who found the [OI] line at $63 \mu \mathrm{m}$ shifted by $100 \mathrm{~km} \mathrm{~s}^{-1}$. Such high velocities, combined with the high densities derived from the non-LTE analysis, indicate that at least part of the atomic emission is generated in dissociative shocks. Indeed, J-shock models predict that the [NeII] intensities observed require shock velocities higher than $70 \mathrm{~km} \mathrm{~s}^{-1}$ (Hollenbach \& McKee 1989). J-shocks are also inferred by water maser emission $183 \mathrm{GHz}$ along the atomic jet axis to the NW (van Kempen et al. 2009, see also Fig. 1). As pointed out in Hollenbach et al. (2013), substantial maser emission in submillimeter wavelengths requires temperatures $\gtrsim 1000 \mathrm{~K}$, which are readily produced behind J-shocks. Such temperatures are consistent with those derived from the [FeII] analysis.

\subsection{On the driving source of the atomic jet}

The evolutionary phase of a protostellar source may be inferred by the mass flux of the ejecta (see Sect. 1). For atomic emission, the mass flux of a jet can be derived using the method described in Dionatos et al. (2009). Summarizing, the method is based on the forbidden atomic line emission being optically thin so that the observed luminosity is proportional to the mass of the emitting gas. It requires knowledge of the velocity of the jet and relies on the assumption that no iron is locked onto dust grains. In the case of L1448-mm, however, which has similar high velocity "bullet" gas as SMM1 (Bachiller et al. 1990; Kristensen et al. 2011), it has been shown that only a fraction between $5 \%$ and $20 \%$ of iron in the outflows is in the gas phase (Dionatos et al. 2009). Here, for the velocity of the atomic jet, we adopt a conservative value of $100 \mathrm{~km} \mathrm{~s}^{-1}$ (Goicoechea et al. 2012) and employ the iron gas-phase fraction estimated in the case of L1448. Based on these assumptions, we find that the two-sided mass flux for the iron is $\sim 2-4 \times 10^{-7} M_{\odot} \mathrm{yr}^{-1}$, consistent for all iron lines. For the adopted velocity, these values correspond to a jet momentum flux of $\sim 5 \times 10^{-5} M_{\odot} \mathrm{km} \mathrm{s}^{-1} \mathrm{yr}^{-1}$, in very good agreement with the values derived for the $\mathrm{CO}$ lobes $\mathrm{Bb} 1$ and $\mathrm{Br} 1$ (Dionatos et al. 2010b). Despite the uncertainties in the derivation of the atomic jet momentum flux here and the CO outflow momentum flux (see Downes \& Cabrit 2007; van der Marel et al. 2013, for extensive discussions), the estimations above suggest that the [FeII] jet has enough thrust to power the large-scale $\mathrm{CO}$ outflow.

[NeII] emission at $12.8 \mu \mathrm{m}$ may be produced through X-ray and FUV irradiation on outflow cavity walls and disk surfaces, or within shocks in protostellar jets. Examining a large sample of Class II protostars, Güdel et al. (2010) demonstrate that sources with jets have one to two orders of magnitude higher [NeII] luminosities compared to sources without jets. The same authors find that the mass loss rate in sources with jets closely correlates with the [NeII] line luminosity. This is also reflected in a decrease by about three orders of magnitude in the [NeII] luminosity between Class I and Class III sources observed in the $\rho$ Oph cloud (Flaccomio et al. 2009). The [NeII] emission detected around SMM1 is clearly extended and closely follows the pattern of the [FeII] jet, so there is little doubt of its origin. The $[\mathrm{NeII}]$ luminosity at the $\mathrm{NW}$ peak ranges from $\sim 9 \times 10^{28}-2 \times 10^{29} \mathrm{erg} \mathrm{s}^{-1}$ for corresponding distances of 260 and $415 \mathrm{pc}$. When compared to the sample of YSO's in $\rho$-Oph (Flaccomio et al. 2009), [NeII] luminosities lie at the border between Class I and Class II sources. For the estimated [NeII] luminosities, the X-wind model calculations of Shang et al. (2010) predict a mass-loss rate of $10^{-7.5}-10^{-7} M_{\odot} \mathrm{yr}^{-1}$, in agreement with the values derived from [FeII].

In conclusion, the momentum flux derived by both $[\mathrm{FeII}]$ and [NeII] lines is $\sim 10^{-5} M_{\odot} \mathrm{km} \mathrm{s}^{-1} \mathrm{yr}^{-1}$, assuming a jet velocity of $100 \mathrm{~km} \mathrm{~s}^{-1}$. Based on the correlation between the momentum flux or force of the outflow and the bolometric luminosity of the source $\left(F_{\mathrm{CO}} / L_{\mathrm{bol}}\right)$ of Cabrit \& Bertout (1992) for Class 0 protostars, SMM1-a with $L_{\text {bol }}=71 L_{\odot}($ Larsson et al. 2000$)$ would be expected to produce a $\mathrm{CO}$ outflow momentum flux of $7 \times 10^{-4} M_{\odot} \mathrm{km} \mathrm{s}^{-1} \mathrm{yr}^{-1}$, almost two orders of magnitude higher than the values estimated here. Consequently, our estimations are too low for a deeply embedded Class 0 protostar and are compatible with a rather evolved Class I/II source (Hartigan et al. 1994; Cabrit 2007). This is in line with the characterization of SMM1-b as a disk source from the SED slope between $7 \mathrm{~mm}$ and $6.9 \mathrm{~cm}$ (Choi 2009), and it provides additional evidence that the atomic jet is indeed driven by the more evolved companion source to the Class 0 protostar SMM1-a.

\section{Conclusions}

We carried out spectro-imaging observations of the region around SMM1 in Serpens with Spitzer/IRS, encompassing a wavelength range between 10 and $38 \mu \mathrm{m}$. These observations trace atomic ([FeII], [NeII], [SI], and [SiII]) and molecular $\left(\mathrm{H}_{2}\right)$ 
emission extending from the protostellar source. In addition, we compared the emission-line maps to deconvolved HiRes IRAC and MIPS images. The main results are summarized as follows.

- The position angles of low- $J \mathrm{H}_{2}$ and atomic emission differ by $\sim 35^{\circ}$. Atomic emission is traced at distances $<5^{\prime \prime}$, while $\mathrm{H}_{2}$ becomes prominent at greater distances from SMM1. Given that pairs of $\mathrm{H}_{2}$ and atomic lines are separated by $\sim 1 \mu \mathrm{m}$ or less, the atomic emission is less extinct and lies in front of the dense envelope surrounding the embedded source SMM1-a. Therefore the atomic emission originates in a companion source lying in the foreground.

- The $\mathrm{H}_{2}$ and atomic emission disentangle the large-scale $\mathrm{CO}$ outflow structure into two outflows. The $\mathrm{H}_{2}$ emission corresponds to the $\mathrm{CO}$ outflow extending roughly in the N-S direction, while the atomic emission drives the high velocity $\mathrm{CO}$ outflow extending in the NW-SE direction. The differentiated outflow morphology is compatible only with a protobinary source.

- LTE and non-LTE analysis of the [FeII] lines suggests an excitation temperature of $\sim 1000 \mathrm{~K}$. Electron densities between $10^{-5}$ and $10^{-6} \mathrm{~cm}^{-3}$ are consistent with the values observed in atomic jets from evolved protostars; however, the temperatures traced by higher excitation [FeII] lines in the near-IR are an order of magnitude higher than the ones traced here.

- The atomic jet has not been traced before in visual or nearIR wavelengths, as it lies behind a dust layer revealed by continuum emission and $\mathrm{CO}_{2}$ absorption bands.

- The mass flux estimated from the [FeII] ranges between $2-4 \times 10^{-7} M_{\odot} \mathrm{yr}^{-1}$ for both sides of the atomic jet. This corresponds to a momentum flux of $\sim 5 \times 10^{-5} M_{\odot} \mathrm{km} \mathrm{s}^{-1} \mathrm{yr}^{-1}$, which agrees with the values corresponding to the NW-SE $\mathrm{CO}$ outflow. Thus the atomic jet has enough thrust to drive the large-scale $\mathrm{CO}$ outflow.

- Compared with X-wind models, the observed [NeII] line luminosity is consistent with mass-loss rates between $10^{-7}$ and $10^{-7.5} M_{\odot} \mathrm{yr}^{-1}$

- Mass-loss rates for both atomic tracers are compatible with measurements for rather evolved Class I/II sources.

- The luminosity of the [NeII] lines compared with the luminosities of protostellar sources in the $\rho$-Oph cloud also confirms that the driving source of the atomic jet is a Class I/II protostar.

The existence of a less embedded companion source to the embedded protostellar source SMM1 has long been suspected (e.g., Eiroa \& Casali 1989; Hodapp 1999; Eiroa et al. 2005; Choi 2009; Dionatos et al. 2010b). The atomic lines detected in this work reveal the ejecta from the binary companion for the first time. Our analysis provides ample evidence that the atomic jet is compatible with a more evolved Class I/II source. Most likely, the companion source driving the atomic jet is SMM1-b. We therefore conclude that SMM1 is a protobinary source, consisting of the known embedded Class 0 protostar SMM1-a (SMM1/FIRS1) and a more evolved Class I/II companion SMM1-b, lying at $\sim 1.5^{\prime \prime}$ to the NW and in the foreground of SMM1-a.

Acknowledgements. We thank the referee (T. Velusamy) for his very constructive comments and for providing the Spitzer HiRes images presented here. This research was supported by a grant from the Instrument Center for Danish Astrophysics (IDA), a Lundbeck Foundation Group Leader Fellowship to JKJ and from the EU FP7-2011 under Grant Agreement No. 284405. This publication is supported by the Austrian Science Fund (FWF). Research at the Centre for Star and Planet Formation is funded by the Danish National Research Foundation and the University of Copenhagen's program of excellence.

\section{References}

Agra-Amboage, V., Dougados, C., Cabrit, S., \& Reunanen, J. 2011, A\&A, 532, A59

Arce, H. G., \& Sargent, A. I. 2006, ApJ, 646, 1070

Bachiller, R., Martin-Pintado, J., Tafalla, M., Cernicharo, J., \& Lazareff, B. 1990, A\&A, 231, 174

Bontemps, S., André, P., Terebey, S., \& Cabrit, S. 1996, A\&A, 311, 858

Cabrit, S. 2007, in Lect. Notes Phys. 723, eds. J. Ferreira, C. Dougados, \& E. Whelan (Berlin: Springer-Verlag), 21

Cabrit, S., \& Bertout, C. 1992, A\&A, 261, 274

Choi, M. 2009, ApJ, 705, 1730

Curiel, S., Rodriguez, L. F., Moran, J. M., \& Canto, J. 1993, ApJ, 415, 191

Davis, C. J., Matthews, H. E., Ray, T. P., Dent, W. R. F., \& Richer, J. S. 1999 , MNRAS, 309, 141

Dionatos, O., Nisini, B., Garcia Lopez, R., et al. 2009, ApJ, 692, 1

Dionatos, O., Nisini, B., Cabrit, S., Kristensen, L., \& Pineau des Forêts, G. 2010a, A\&A, 521, A7

Dionatos, O., Nisini, B., Codella, C., \& Giannini, T. 2010b, A\&A, 523, A29

Dionatos, O., Jørgensen, J. K., Green, J. D., et al. 2013, A\&A, 558, A88

Downes, T. P., \& Cabrit, S. 2007, A\&A, 471, 873

Dzib, S., Loinard, L., Mioduszewski, A. J., et al. 2010, ApJ, 718, 610

Eiroa, C., \& Casali, M. M. 1989, A\&A, 223, L17

Eiroa, C., Torrelles, J. M., Curiel, S., \& Djupvik, A. A. 2005, AJ, 130, 643

Enoch, M. L., Corder, S., Dunham, M. M., \& Duchêne, G. 2009, ApJ, 707, 103

Ferreira, J. 1997, A\&A, 319, 340

Flaccomio, E., Stelzer, B., Sciortino, S., et al. 2009, A\&A, 505, 695

Goicoechea, J. R., Cernicharo, J., Karska, A., et al. 2012, A\&A, 548, A77

Goldsmith, P. F., \& Langer, W. D. 1999, ApJ, 517, 209

Green, J. D., Evans, II, N. J., Jørgensen, J. K., et al. 2013, ApJ, 770, 123

Güdel, M., Lahuis, F., Briggs, K. R., et al. 2010, A\&A, 519, A113

Gueth, F., \& Guilloteau, S. 1999, A\&A, 343, 571

Hartigan, P., Morse, J. A., \& Raymond, J. 1994, ApJ, 436, 125

Hartigan, P., Edwards, S., \& Ghandour, L. 1995, ApJ, 452, 736

Hodapp, K. W. 1999, AJ, 118, 1338

Hogerheijde, M. R., van Dishoeck, E. F., Salverda, J. M., \& Blake, G. A. 1999, ApJ, 513, 350

Hollenbach, D., \& McKee, C. F. 1989, ApJ, 342, 306

Hollenbach, D., Elitzur, M., \& McKee, C. F. 2013, ApJ, 773, 70

Houck, J. R., Roellig, T. L., van Cleve, J., et al. 2004, ApJS, 154, 18

Kristensen, L. E., van Dishoeck, E. F., Tafalla, M., et al. 2011, A\&A, 531, L1

Lahuis, F., van Dishoeck, E. F., Jørgensen, J. K., Blake, G. A., \& Evans, N. J. 2010, A\&A, 519, A3

Larsson, B., Liseau, R., Men'shchikov, A. B., et al. 2000, A\&A, 363, 253

Neufeld, D. A., Melnick, G. J., Sonnentrucker, P., et al. 2006, ApJ, 649, 816

Neufeld, D. A., Nisini, B., Giannini, T., et al. 2009, ApJ, 706, 170

Nisini, B., Caratti o Garatti, A., Giannini, T., \& Lorenzetti, D. 2002, A\&A, 393, 1035

Panoglou, D., Cabrit, S., Pineau des Forêts, G., et al. 2012, A\&A, 538, A2

Ralchenko, Y., Kramida, A. E., Reader, J., \& the NIST ASD Team 2011, available: http://physics.nist.gov/asd, 309, 1

Shang, H., Glassgold, A. E., Lin, W.-C., \& Liu, C.-F. J. 2010, ApJ, 714, 1733

Shu, F., Najita, J., Ostriker, E., et al. 1994, ApJ, 429, 781

Smith, J. D. T., Armus, L., Dale, D. A., et al. 2007, PASP, 119, 1133

Straižys, V., Černis, K., \& Bartašiūtè, S. 2003, A\&A, 405, 585

Takami, M., Chrysostomou, A., Ray, T. P., et al. 2004, A\&A, 416, 213

Testi, L., \& Sargent, A. I. 1998, ApJ, 508, L91

van der Marel, N., Kristensen, L. E., Visser, R., et al. 2013, A\&A, 556, A76

van der Tak, F. F. S., Black, J. H., Schöier, F. L., Jansen, D. J., \& van Dishoeck, E. F. 2007, A\&A, 468, 627

van Kempen, T. A., Wilner, D., \& Gurwell, M. 2009, ApJ, 706, L22

Velusamy, T., Langer, W. D., \& Marsh, K. A. 2007, ApJ, 668, L159

Velusamy, T., Marsh, K. A., Beichman, C. A., Backus, C. R., \& Thompson, T. J. 2008, AJ, 136, 197

Velusamy, T., Langer, W. D., Kumar, M. S. N., \& Grave, J. M. C. 2011, ApJ, 741,60

Velusamy, T., Langer, W. D., \& Thompson, T. 2014, ApJ, 783, 6

Visser, R., Kristensen, L. E., Bruderer, S., et al. 2012, A\&A, 537, A55

White, G. J., Casali, M. M., \& Eiroa, C. 1995, A\&A, 298, 594

Wiese, J. R., \& Fuhr, W. L. 2007, J. Phys. Chem. Ref. Data, 35, 1669

Winston, E., Megeath, S. T., Wolk, S. J., et al. 2007, ApJ, 669, 493

Wu, Y., Wei, Y., Zhao, M., et al. 2004, A\&A, 426, 503

Yıldız, U. A., Kristensen, L. E., van Dishoeck, E. F., et al. 2013, A\&A, 556, A89

Zhang, H. L., \& Pradhan, A. K. 1995, A\&A, 293, 953

Zhang, Q., Hunter, T. R., Brand, J., et al. 2005, ApJ, 625, 864 